\title{
Incidence of tricuspid regurgitation and the total number of tricuspid valve surgery in patients with mitral valve disease at Clinical Hospital Center Osijek
}

\author{
Sandra Makarović*, Zorin Makarović, Marija Maras, Andrea Džumhur, Dragan Novosel \\ Clinical Hospital Center Osijek, Osijek, Croatia
}

Background: Pathological tricuspid regurgitation is often secondary, resulting from the left-sided heart disease. The need for correction of tricuspid regurgitation is usually considered at the time of surgical correction of the left-sided valve lesions

Methods: We examined the incidence of tricuspid regurgitation in 60 patients with mitral valve disease, indicated for mitral valve surgery in our center, in the period from 2009 to 2012. We evaluated which of these accompanying conditions follow severe tricuspid regurgitation (atrial fibrillation, huge left atrium, dilated tricuspid annulus, left ventricular dysfunction).

Results: The results showed that $33 \%$ of all patients undergoing mitral valve surgery had tricuspid valve surgery. $65 \%$ of them had severe and $35 \%$ had moderate tricuspid regurgitation. $38 \%$ of all patients (with and without tricuspid valve surgery) had moderate tricuspid regurgitation; $31 \%$ of patients with moderate tricuspid regirgitation had tricuspid valve surgery, $69 \%$ of them did not have tricuspid valve surgery. In patients with severe tricuspid regurgitation we found higher frequency of atrial fibrillation ( $p=0.013)$, tricuspid an- nular dilatation $(p=0.002)$, and right atrial dilatation $(p=0.013)$. There were no higher frequency of left ventricular dysfunction $(p=0.405)$, left atrial dilatation $(p=0.166)$, or estimated sistolic right ventricle pressure $(p=0.998)$ in patients with tricuspid regurgitation.

Conclusion: Significant tricuspid regurgitation is present in more than one-third of patients with mitral valve disease. Tricuspid annular dilatation, right atrial dilatation and atrial fibrillation is more frequent in patients with severe tricuspid regurgitation.

KEYWORDS: tricuspid regurgitation, tricuspid valve surgery, mitral valve disease.

Received: $20^{\text {th }}$ Mar 2013

*Address for correspondence: Clinical Hospital Center Osijek, J. Huttlera 4, HR31000 Osijek, Croatia.

Phone: +385-31-511-511

E-mail: sandramakarovic@yahoo.com

\section{Literature}

1. Shiran A, Sagie A. Tricuspid regurgitation in mitral valve disease. Incidence, prognostic implications, mechanism, and management. J Am Coll Cardiol. 2009;53:401-8. 2. Colombo T, Russo C, Ciliberto GR, Lanfranconi M, Bruschi G, Agati S, et al. Tricuspid regurgitation secondary to mitral valve disease: tricuspid annulus function as guide to tricuspid valve repair. Cardiovas Surg. 2001;9:369-77. 